\title{
STABILITY ANALYSIS FOR CONSTRAINED PRINCIPAL AXIS SLEW MANEUVERS
}

\author{
Hans Seywald* \\ Analytical Mechanics Associates \\ Hampton VA \\ seywaldescb2. larc. nasa.gov
}

\author{
Kyong B. $\operatorname{Lim}^{\dagger}$ \\ NASA Langley Research Center \\ Hampton VA \\ k.b.lim@larc.nasa.gov
}

\author{
Tobin C. Anthony ${ }^{\ddagger}$ \\ NASA Goddard Space Flight Center \\ Greenbelt, MD \\ tobin .anthony@gsf c.nasa.gov
}

\begin{abstract}
This paper addresses the problem of reorienting a rigid spacecraft from arbitrary initial conditions to prescribed final conditions with zero angular velocity. The control law analyzed is based on quaternion feedback and leaves the user to choose two gains as functions of position, angular rate, and time. For arbitrary initial states, conditions on the controller gains are identified that guarantee global asymptotic stability. For the special case of rest-to-rest reorientations, the control law reduces to earlier results involving a principal axis rotation. The paper also addresses slew rate constraints, both, in terms of the two and infinity norms.
\end{abstract}

\section{Introduction and Literature Survey}

Spacecraft reorientation problems have been treated extensively in the technical literature [1][8]. Open-loop approaches enable the calculation of high-precision solutions that minimize a userprescribed cost index such as fuel consumption or maneuver time. However, these approaches usually involve iterative procedures and are hence, in most cases, computationally too expensive and unreliable for on-board implementation.

Closed-loop or feedback approaches perform only noniterative procedures and calculate the current control action based on the current state and time. Feedback approaches typically perform at best nearoptimally, and for nonlinear controllers and/or non-

${ }^{*}$ Staff Scientist, 17 Research Drive, Hampton, VA 23666, Member AIAA.

${ }^{\dagger}$ Research Engineer, Guidance and Control Branch, Flight Dynamics and Control Division

${ }^{\ddagger}$ Aerospace Engineer, Guidance, Navigation, and Control Group, Member AIAA. linear dynamical systems it is highly nontrivial to guarantee that the controller always drives the states to the desired position. Additionally, it is usually difficult to enforce state constraints, such as slew rate limits.

In practice, slew rate limits often arise from the requirement not to exceed the capabilities of a spacecraft inertial reference unit (IRU) to sense angular velocity; slewing too fast can saturate the IRU thereby limiting the controller's ability to track the maneuver. Depending on the spacecraft's architecture and the involved on-board instrumentation these physical constraints are best captured by twonorm or infinity-norm constraints on the angular position.

The general nonlinear feedback control law analyzed in this paper has been in service in one form or other for many years in numerous applications of near-minimum-time satellite reorientation[9]-[11]. It is derived through feedback linearization applied to a representation of the dynamical system in terms of quaternions in favor of Euler angles. The paper completely analyzes this controller in terms of its physical properties and identifies the conditions on the controller gains required to guarantee global asymptotic stability and satisfaction of slew rate constraints. Thus, the paper provides a frame work of conditions within which the control engineer can optimize the control gain functions' remaining degrees of freedom without having to consider global stability issues and satisfaction of slew rate limits.

In [12], Wie and Lu have investigated the constrained slew controller implemented on the XTE spacecraft[10]. This controller represents a special case of the general controller analyzed in the present paper. However, Wie and Lu only investigated restto-rest maneuvers and assumed perfect execution of all control commands without regard to exter- 
nal perturbations. For this special case, the reorientation maneuver reduces to a one-degree-of-freedom principal axis rotation. The intent of this paper is to provide a more comprehensive look at the stability of the constrained eigenaxis slew maneuver.

\section{Problem Formulation}

Consider the attitude dynamics of a rigid spacecraft. The equations of motion can be represented in the following form:

$$
\begin{aligned}
J \dot{\omega} & =-\omega \times J \omega+u \\
\dot{q} & =-\frac{1}{2} \omega \times q+\frac{1}{2} q_{4} \omega \\
\dot{q}_{4} & =-\frac{1}{2} \omega^{T} q .
\end{aligned}
$$

Here, $J$ denotes a constant $3 \times 3$-inertia matrix, $\omega^{T}=\left[\omega_{1}, \omega_{2}, \omega_{3}\right]$ denotes the angular velocity vector, $u^{T}=\left[u_{1}, u_{2}, u_{3}\right]$, denotes the control torque input vector, and $q^{T}=\left[q_{1}, q_{2}, q_{3}\right]$ together with $q_{4}$ represent the angular position through its associated quaternion vector $\left[q^{T}, q_{4}\right]$.

Euler's rotational theorem states that the rigidbody attitude can be changed from any given orientation to any other orientation by rotating the body about an axis, called the Euler axis, that is fixed, both, with respect to the inertial frame as well as with respect to the rigid body. For a given reorientation, let $e^{T}=\left[e_{1}, e_{2}, e_{3}\right]$ denote a unit vector along the Euler axis, and let $\theta$ denote the associated rotation angle about the Euler axis. Then the quaternion vector $\left[q^{T}, q_{4}\right]$ satisfies

$$
\begin{aligned}
& q_{1}=e_{1} \sin (\theta / 2) \\
& q_{2}=e_{2} \sin (\theta / 2) \\
& q_{3}=e_{3} \sin (\theta / 2) \\
& q_{4}=\cos (\theta / 2)
\end{aligned}
$$

By construction, the quaternion vector $\left[q^{T}, q_{4}\right]$ is always of norm one, i.e.

$$
q_{1}^{2}+q_{2}^{2}+q_{3}^{2}+q_{4}^{2}=1 .
$$

The vector $\left[q^{T}, q_{4}\right]$ has no physical meaning unless constraint (8) is satisfied. It is important to note that constance of the norm of $\left[q^{T}, q_{4}\right]$ is guaranteed through the equations of motion (1)-(3), irrespective of the chosen control $u$. Hence, $(8)$ is guaranteed to be satisfied (up to the precision of the numerical integration ) if it is satisfied at a single point, say, at the initial time.

We consider the following problem:
Definition 1 Given the equations of motion (1)(3) and a positive constant $\omega_{\max }$, find a feedback control law

$$
u=f\left(\omega, q, q_{4}, t\right)
$$

that drives the states $\omega, q, q_{4}$ from arbitrary initial conditions $\omega\left(t_{0}\right) \in \mathbf{R}^{3}, q\left(t_{0}\right) \in \mathbf{R}^{3}, q_{4}\left(t_{0}\right) \in \mathbf{R}$ with $q\left(t_{0}\right)^{T} q\left(t_{0}\right)+q_{4}\left(t_{0}\right)^{2}=1$ to $\omega=0, q=0, q_{4}= \pm 1$, such that the state constraints

$$
\|\omega(t)\|_{2} \leq \omega_{\max }
$$

and/or

$$
\|\omega(t)\|_{\infty} \leq \omega_{\max }
$$

remain satisfied for all times.

The symbols, $\|\cdot\|_{2}$ and $\|\cdot\|_{\infty}$ denote the Euclidean and $\infty$ norms in the corresponding finite dimensional vector space, respectively, i.e.

$$
\|\omega(t)\|_{2}=\sqrt{\omega_{1}(t)^{2}+\omega_{2}(t)^{2}+\omega_{3}(t)^{2}}
$$

and

$$
\|\omega(t)\|_{\infty}=\max \left\{\left|\omega_{1}(t)\right|,\left|\omega_{2}(t)\right|,\left|\omega_{3}(t)\right|\right\}
$$

\section{$\underline{\text { Eigenaxis Rotation Controller }}$}

In the present paper, a controller (9) of the form

$$
\begin{aligned}
u\left(\omega, q, q_{4}, t\right)= & \omega \times J \omega \\
& -k\left(\omega, q, q_{4}, t\right) J q \\
& -c\left(\omega, q, q_{4}, t\right) J \omega
\end{aligned}
$$

is investigated, where $k$ and $c$ denote scalar functions of their arguments $\omega, q, q_{4}$, and $t$, respectively. This form of the controller is motivated by the desire to eliminate the cross product term in (1) as well as a state feedback. Explicitly, inserting (12) back into (1) yields

$$
\dot{\omega}=-k q-c \omega
$$

so that (12) can be interpreted also as feedback linearization if $k$ and $c$ do not explicitly depend on states.

In the following, we will prove a number of useful properties of the controller (12).

Property 1 Consider the dynamical system (1)(3) with the feedback controller (12), where $k$ and $c$ are Lipschitz-bounded with respect to $\omega, q, q_{4}$, and piecewise continuous with respect to $t$. Then $\omega(t)$, $q(t), \omega(0)$ and $q(0)$ are collinear if and only if $\omega(0)$ and $q(0)$ are collinear. 


\section{Proof of Property 1:}

$\Rightarrow$ ) Assume $\omega(t), q(t), \omega(0)$ and $q(0)$ are collinear for all times. Then, obviously, $\omega(0)$ and $q(0)$ are collinear.

$\Longleftarrow$ ) Assume $\omega(0)$ and $q(0)$ are collinear. We first show that $\omega(t) \times q(t) \equiv 0$ for all times. We have

$$
\frac{d}{d t}(\omega \times q)=-c \omega \times q-\frac{1}{2} \omega \times(\omega \times q) .
$$

Here we used $q \times q=0$ and $\omega \times \omega=0$. Now consider the system of ordinary differential equations consisting of the equations (2), (3), (13), and (14). It can be verified that the right-hand side of this system is Lipschitz-bounded with respect to the states $q, q_{4}, \omega$, and $(\omega \times q)$, and independent of time $t$ (thus measurable with respect to time $t$.) Hence, whenever a solution to the system (2), (3), (13), and (14) exists for given initial conditions, this solution is determined uniquely (see the Appendix I of $[13])$. As $(\omega \times q) \equiv 0$ obviously furnishes a solution to equation (14) subject to the initial condition $(\omega \times q)(0)=0$ it is clear that $\omega \times q$ is guaranteed to remain identically zero for all solutions to the system (2), (3), (13), whenever the initial conditions are such that $\omega(0) \times q(0)=0$. Hence $\omega(t)$, and $q(t)$ are guaranteed to remain collinear whenever $\omega(0)$ and $q(0)$ are.

To show that $\omega(t)$ and $q(t)$ are collinear not only with respect to each other but also with respect to $q(0)$, let us define the new states

$$
\begin{aligned}
& \Omega(t)=\omega(t) \times q(0) \\
& Q(t)=q(t) \times q(0) .
\end{aligned}
$$

Then, using the fact that $\omega(t) \times q(t)$ is identically zero, it can be verified that $\Omega$ and $Q$ satisfy the following differential equations:

$$
\begin{aligned}
& \dot{\Omega}(t)=-k Q(t)-c \Omega(t) \\
& \dot{Q}(t)=\frac{1}{2} q_{4}(t) \Omega(t) .
\end{aligned}
$$

Considering the augmented system of differential equations consisting of equations (2), (3), (13), and (16), and using arguments similar to the ones used above to show that $\omega(t) \times q(t)$ is identically zero, we can show that $\Omega$ and $Q$ remain identically zero whenever they are zero at initial time. This implies that $\omega(t)$ and $q(t)$ are collinear to $q(0)$ if $\omega(0)$ is collinear to $q(0)$.

By virtue of the fact that $\omega(t)$ and $q(t)$ are collinear for all times if $\omega(0)$ and $q(0)$ are, the previous result implies that $\omega(t)$ and $q(t)$ are collinear also to $\omega(0)$ if $\omega(0)$ is collinear to $q(0)$.

q.e.d.
The statement of Property 1 can be strengthened by replacing "piecewise continuous with respect to time $t$ " through "measurable with respect to time t". Loosely speaking, the set of measurable functions includes all piecewise continuous functions, plus some functions with an infinite number of discontinuous jumps. For a more precise characterization of measurable functions, see the Appendix I of [13], or Section 3.7 of [14]. Note that by definition, a function $f(x)$ is Lipschitz-bounded with respect to $x$ if and only if for every $x$ in its domain there is a constant $L$ such that $\|f(x+\Delta x)-f(x)\|_{2} \leq L\|\Delta x\|_{2}$ for all sufficiently small $\Delta x$. Hence, the condition of Lipschitz-boundedness in Property 1 is guaranteed to be satisfied if the functions $c$ and $k$ are differentiable with respect to $\omega, q, q_{4}$, and have uniformly bounded gradients for all fixed $t$.

Property 1 shows that $\omega(0), q(0), \omega(t)$, and $q(t)$ remain collinear for all times if $\omega(0)$ and $q(0)$ are collinear. In this case the body performs a rotation about an axis that remains fixed in inertial space. The condition that $\omega(0)$ and $q(0)$ be collinear is trivially satisfied for a rest-to-rest reorientation where $\omega(0)=0$.

Next we want to investigate what happens if the control law in Property 1 is applied to a system where $\omega(0)$ and $q(0)$ are not collinear. Note that this situation can arise as a result of external perturbations; it is important that any "out-of-plane" component does not grow with time.

Property 2 Consider the system (1)-(3) with the control law (12). Now assume there is a constant $c_{\min }$ such that

$$
c\left(\omega, q, q_{4}, t\right) \geq c_{\min } \forall \omega, q, q_{4}, t .
$$

Then

$$
\|\omega \times q\|_{2} \leq\left\|\omega\left(t_{0}\right) \times q\left(t_{0}\right)\right\|_{2} \cdot e^{-c_{m i n}\left(t-t_{0}\right)} .
$$

PRoOF: We have

$$
\begin{aligned}
& \frac{d}{d t}\left(\|\omega \times q\|_{2}^{2}\right) \\
& \stackrel{(14)}{=} 2(\omega \times q)^{T}\left[-c \omega \times q-\frac{1}{2} \omega \times(\omega \times q)\right] \\
& =-2 c(\omega \times q)^{T}(\omega \times q)-(\omega \times q)^{T}[\omega \times(\omega \times q)]
\end{aligned}
$$

The second term on the right-hand side is zero as $(\omega \times q)^{T}$ is normal to $\omega \times(\omega \times q)$. Hence

$$
\begin{aligned}
\frac{d}{d t}\left(\|\omega \times q\|_{2}^{2}\right) & =-2 c \cdot(\omega \times q)^{T}(\omega \times q) \\
& =-2 c\|\omega \times q\|_{2}^{2}
\end{aligned}
$$


With the lower bound (17) on $c$, this yields

$$
\|\omega \times q\|_{2}^{2} \leq\left\|\omega\left(t_{0}\right) \times q\left(t_{0}\right)\right\|_{2}^{2} \cdot e^{-2 c_{m i n}\left(t-t_{0}\right)},
$$

which implies (18).

q.e.d.

Property 2 states that the normal component $\omega \times$ $q$ decays exponentially in norm if and only if the constant $c_{\text {min }}$ in (17) satisfies

$$
c_{\min }>0 .
$$

\section{Global Stability Analysis}

Property 3 Consider the equations of motion (1)(3) with the control law (12). Assume that

A1) the gains $k$ and $c$ satisfy the conditions stated in Properties 1 and 2 , with $c_{\text {min }}>0$,

AD) $k$ is a positive constant, and

A3) $c$ is bounded in the sense that for every $M \in$ $\mathbf{R}$ there is a $C(M) \in \mathbf{R}$ such that $\left|c\left(\omega, q, q_{4}, t\right)\right| \leq$ $C(M)$ as long as $\|\omega\|_{2} \leq M$. Then,

P1) $\lim _{t \rightarrow \infty}\|\omega(t)\|_{2}=0$

P2) $\lim _{t \rightarrow \infty} q_{4}(t)=+1$ or -1 ,

P3) $\lim _{t \rightarrow \infty}\|q(t)\|_{2}=0$.

Proof: Define the function

$$
V=\frac{1}{4 k}\left(\omega_{1}^{2}+\omega_{2}^{2}+\omega_{3}^{2}\right)-q_{4}
$$

Differentiating $V$ and inserting (1), (2), (3), (12) yields after some calculation

$$
\dot{V}=-\frac{c}{2 k} \omega^{T} \omega \leq 0 .
$$

Assume property P2 is not satisfied. Since $V$ is monotonically decreasing and bounded from below this implies that there is a real number $d \neq \pm 1$ such that $V$ converges to $d$ as time goes to infinity. Let $d_{0}$ denote the minimum distance of $d$ to \pm 1 , i.e.

$$
d_{0}=\min \{|d-1|,|d+1|\}>0 .
$$

Then let us pick positive real numbers $\Omega, \omega_{0}$, and $\epsilon_{0}$ such that

$$
\begin{aligned}
\Omega & \geq \sqrt{4 k\left(V\left(t_{0}\right)+1\right)} \\
\omega_{0} & \leq \frac{1}{2} \\
\omega_{0} & \leq \sqrt{k d_{0}}
\end{aligned}
$$

$$
\begin{aligned}
\omega_{0} & \leq \frac{1}{2}\left[k\left(\frac{2}{k \frac{d_{0}}{2}}\right)^{2}+C(\Omega)\left(\frac{2}{k \frac{d_{0}}{2}}\right)\right]^{-1} \\
\epsilon_{0} & \leq \frac{d_{0}}{4} \\
\epsilon_{0} & \leq \frac{\frac{c_{\text {min }}}{2 k}\left(\frac{\omega_{0}}{2}\right)^{2} \omega_{0}}{4(k+C(\Omega) \Omega)}
\end{aligned}
$$

As, by assumption, $V$ approaches $d$ arbitrarily closely as time goes to infinity, we can pick a time $t_{0}$ such that

$$
d \leq V \leq d+\epsilon_{0} \quad \forall t \geq t_{0} .
$$

The assumption that property $\mathrm{P} 2$ is violated $(\Rightarrow$ $q_{4}(t)$ does not go to $\pm 1 \Rightarrow\|q(t)\|_{2}$ does not go to zero) implies that we can pick $t_{0}$ such that, in addition to $(29), q\left(t_{0}\right) \neq 0$, and we can define

$$
\begin{aligned}
& T_{1}=\frac{\omega_{0}}{2(k+C(\Omega) \Omega)} \\
& T_{2}=\frac{2}{k\left\|q\left(t_{0}\right)\right\|_{2}}
\end{aligned}
$$

Solving (20) for $\omega^{T} \omega$ and taking the square root we can show immediately that $\Omega$ furnishes an upper bound on $\|\omega\|_{2}$ for all $t \geq t_{0}$, namely

$$
\begin{aligned}
\|\omega\|_{2} & =\sqrt{4 k\left(V(t)+q_{4}(t)\right)} \\
& \leq \sqrt{4 k\left(V\left(t_{0}\right)+1\right)} \\
& \leq \Omega
\end{aligned}
$$

The second line in (32) follows from (29), which implies $V\left(t_{0}\right) \leq v(t)$, and from $\left|q_{4}\right| \leq 1$. The third line follows from (23). We have to consider two cases, namely $\left\|\omega\left(t_{0}\right)\right\|_{2} \geq \omega_{0}$ and $\left\|\omega\left(t_{0}\right)\right\|_{2}<\omega_{0}$.

Case 1: Assume

$$
\left\|\omega\left(t_{0}\right)\right\|_{2} \geq \omega_{0} .
$$

From equation (1), with $u$ replaced by the righthand side of (12) we find that for all $t \geq t_{0}$

$$
\begin{aligned}
\|\dot{\omega}(t)\|_{2} & =\|-k q(t)-c \omega(t)\|_{2} \\
& \leq k\|q(t)\|_{2}+c\|\omega(t)\|_{2} \\
& \leq k+C(\Omega) \Omega
\end{aligned}
$$

On the interval from $t_{0}$ to $t_{0}+T_{1}$ we hence have

$$
\omega(t)=\omega\left(t_{0}\right)+\Delta \omega(t)
$$

with

$$
\begin{aligned}
\|\Delta \omega(t)\|_{2} & \leq(k+C(\Omega) \Omega) T_{1} \\
& =\frac{\omega_{0}}{2}
\end{aligned}
$$


Hence, for all $t \in\left[t_{0}, t_{0}+T_{1}\right]$

$$
\begin{aligned}
\|\omega(t)\|_{2} & =\left\|\omega\left(t_{0}\right)+\Delta \omega(t)\right\|_{2} \\
& \geq\left\|\omega\left(t_{0}\right)\right\|_{2}-\|\Delta \omega(t)\|_{2} \\
& \geq \omega_{0}-\frac{\omega_{0}}{2} \\
& =\frac{\omega_{0}}{2} .
\end{aligned}
$$

Hence

$$
\begin{aligned}
V\left(t_{0}+T_{1}\right) & \stackrel{(21)}{=} V\left(t_{0}\right)-\int_{t_{0}}^{t_{0}+T_{1}} \frac{c}{2 k} \omega^{T} \omega d t \\
& \leq V\left(t_{0}\right)-\frac{c_{\min }}{2 k}\left(\frac{\omega_{0}}{2}\right)^{2} T_{1} \\
& \stackrel{(28)}{\leq} V\left(t_{0}\right)-2 \epsilon_{0} .
\end{aligned}
$$

But, assuming that $V\left(t_{0}\right)$ satisfies (29), (38) implies that $V\left(t_{0}+T_{1}\right)$ violates $(29)$. Hence case 1 is not possible.

Case 2: Assume

$$
\left\|\omega\left(t_{0}\right)\right\|_{2}<\omega_{0} .
$$

If $\|\omega(t)\|_{2}$ becomes greater or equal $\omega_{0}$ at some time $t_{1}>t_{0}$, then case 2 reduces to case 1 with $t_{0}$ replaced by $t_{1}$. Hence, we only have to consider the case where

$$
\|\omega(t)\|_{2}<\omega_{0} \quad \forall t \geq t_{0} .
$$

Solving equation (20) for $q_{4}-1$, we find that for all $t \geq t_{0}$

$$
\begin{aligned}
&\left|q_{4}-1\right|=\left|\frac{\omega^{T} \omega}{4 k}-V-1\right| \\
&=\left|\frac{\omega^{T} \omega}{4 k}-(V-d)-(d+1)\right| \\
& \geq \quad-\left|\frac{\omega^{T} \omega}{4 k}\right|-|V-d|+|d+1| \\
& \stackrel{(40),(29),(22)}{\geq}-\frac{\omega_{0}^{2}}{4 k}-\epsilon_{0}+d_{0} \\
& \stackrel{(25),(27)}{\geq} \frac{d_{0}}{2}
\end{aligned}
$$

and similarly

$$
\begin{aligned}
&\left|q_{4}+1\right|=\left|\frac{\omega^{T} \omega}{4 k}-V+1\right| \\
&=\left|\frac{\omega^{T} \omega}{4 k}-(V-d)-(d-1)\right| \\
& \geq \quad-\left|\frac{\omega^{T} \omega}{4 k}\right|-|V-d|+|d-1| \\
& \stackrel{(40),(29),(22)}{\geq}-\frac{\omega_{0}^{2}}{4 k}-\epsilon_{0}+d_{0} \\
& \stackrel{(27),(25)}{\geq} \frac{d_{0}}{2}
\end{aligned}
$$

Hence, from (41), (42)

$$
\left|q_{4}-1\right| \cdot\left|q_{4}+1\right| \geq\left(\frac{d_{0}}{2}\right)^{2}
$$

for all $t \geq t_{0}$. Noting that $\left|q_{4}-1\right| \cdot\left|q_{4}+1\right|=\left|q_{4}^{2}-1\right|=$ $q^{T} q,(43)$ immediately yields

$$
\|q(t)\|_{2} \geq \frac{d_{0}}{2} \quad \forall t \geq t_{0} .
$$

From (2) we find now that for all $t \geq t_{0}$

$$
\begin{aligned}
\|\dot{q}\|_{2} & =\left\|-\frac{1}{2} \omega \times q+\frac{1}{2} q_{4} \omega\right\|_{2} \\
& \leq \frac{1}{2} \underbrace{\|\omega\|_{2}}_{\leq \omega_{0}} \underbrace{\|q\|_{2}}_{\leq 1}+\frac{1}{2} \underbrace{\left|q_{4}\right|}_{\leq 1} \underbrace{\|\omega\|_{2}}_{\leq \omega_{0}} \\
& \leq \omega_{0},
\end{aligned}
$$

so that on the interval from $t_{0}$ to $t_{0}+T_{2}$

$$
q(t)=q\left(t_{0}\right)+\Delta q(t)
$$

with

$$
\begin{aligned}
\|\Delta q(t)\|_{2} & =\left\|\int_{t_{0}}^{t_{0}+T_{2}} \dot{q}(t) d t\right\|_{2} \\
& \leq \int_{t_{0}}^{t_{0}+T_{2}}\left\|_{2} \dot{q}(t)\right\|_{2} d t \\
& \leq \omega_{0} T_{2} .
\end{aligned}
$$

Using (46), (47) in equation (1) with $u$ replaced by the right-hand side of (12) yields on the interval $\left[t_{0}, t_{0}+T_{2}\right]$

$$
\begin{aligned}
\dot{\omega}(t) & =-k q(t)-c \omega(t) \\
& =-k q\left(t_{0}\right)+\Delta \dot{\omega}
\end{aligned}
$$

where

$$
\begin{aligned}
\|\Delta \dot{\omega}\|_{2} & =\|-k \Delta q(t)-c \omega(t)\|_{2} \\
& \leq k\|\Delta q(t)\|_{2}+c\|\omega(t)\|_{2} \\
\stackrel{(40),(47)}{\leq} & k \omega_{0} T_{2}+C\left(\omega_{0}\right) \omega_{0} \\
& \stackrel{(32)}{\leq} \quad k \omega_{0} T_{2}+C(\Omega) \omega_{0}
\end{aligned}
$$

From (48), (49), we see that on the interval $t \in$ $\left[t_{0}, t_{0}+T_{2}\right]$

$$
\omega(t)=\omega\left(t_{0}\right)-k q\left(t_{0}\right)\left(t-t_{0}\right)+\Delta \omega(t)
$$

where

$$
\begin{aligned}
& \|\Delta \omega(t)\|_{2} \\
& \quad \leq\left(k \omega_{0} T_{2}+C(\Omega) \omega_{0}\right) T_{2}
\end{aligned}
$$




$$
\begin{aligned}
& =\omega_{0}\left(k T_{2}^{2}+C(\Omega) T_{2}\right) \\
& \stackrel{(31)}{=} \omega_{0}\left[k\left(\frac{2}{k\left\|q\left(t_{0}\right)\right\|_{2}}\right)^{2}+C(\Omega)\left(\frac{2}{k\left\|q\left(t_{0}\right)\right\|_{2}}\right)\right] \\
& \stackrel{(44)}{\leq} \omega_{0}\left[k\left(\frac{2}{k \frac{d_{n}}{2}}\right)^{2}+C(\Omega)\left(\frac{2}{k \frac{d_{n}}{2}}\right)\right] \\
& \stackrel{(26)}{\leq} \frac{1}{2}
\end{aligned}
$$

From (50) we now find

$$
\begin{aligned}
& \left\|\omega\left(t_{0}+T_{2}\right)\right\|_{2} \\
& =\quad\left\|\omega\left(t_{0}\right)-k q\left(t_{0}\right) T_{2}+\Delta \omega\left(t_{0}+T_{2}\right)\right\|_{2} \\
& \geq \quad-\left\|\omega\left(t_{0}\right)\right\|_{2}+k\left\|q\left(t_{0}\right)\right\|_{2} T_{2} \\
& -\left\|\Delta \omega\left(t_{0}+T_{2}\right)\right\|_{2} \\
& \stackrel{(39),(31),(51)}{\geq}-\omega_{0}+k\left\|q\left(t_{0}\right)\right\|_{2} \frac{2}{k\left\|q\left(t_{0}\right)\right\|_{2}}-\frac{1}{2} \\
& \stackrel{(24)}{\geq}-\frac{1}{2}+2-\frac{1}{2} \\
& =1
\end{aligned}
$$

But, in light of (24), (52) implies that $\| \omega\left(t_{0}+\right.$ $\left.T_{2}\right) \|_{2} \geq \omega_{0}$, which contradicts (40). Hence case 2 can be excluded, which completes the proof of property P2.

Property P3 follows immediately from Property P2 and $q^{T} q+q_{4}^{2}=1$ (see equation (8)).

Now assume Property P1 is not satisfied. As $q_{4} \rightarrow$ \pm 1 (property P2) and $\dot{V} \leq 0$ (see equation (21)) this assumption implies that there is a real number $d>0$ and a time $t_{1}$ such that $\|\omega(t)\|_{2}>d$ for all times $t \geq t_{1}$. With (21) this implies this implies that $V$ is unbounded from below. But this contradicts Property P2. Hence $\|\omega(t)\|_{2} \rightarrow 0$ as $t \rightarrow \infty$.

q.e.d.

It is interesting to note that basically the same results as those obtained in Property 3 can be derived if $k$ is chosen negative instead of positive. Using

$$
V=-\frac{1}{4 k}\left(\omega_{1}^{2}+\omega_{2}^{2}+\omega_{3}^{2}\right)+q_{4}
$$

instead of the function $V$ in (20), the proof of this proposition is analogous to the proof of Property 3 . In summary, Property 3 can hence be replaced by the following stronger statement:

Property 4 Consider the equations of motion (1)(3) with the control law (12). Assume that

A1) the gains $k$ and $c$ satisfy the conditions stated in Properties 1 and 2 , with $c_{\min }>0$,

A2) $k$ is a nonzero constant, and
A3) $c$ is bounded in the sense that for every $M \in$ $\mathbf{R}$ there is a $C(M) \in \mathbf{R}$ such that $\left|c\left(\omega, q, q_{4}, t\right)\right| \leq$ $C(M)$ as long as $\|\omega\|_{2} \leq M$.

Then, as time $t$ goes to infinity, we have

P1) $\|\omega(t)\|_{2}$ approaches 0 ,

$P$ 2) $q_{4}(t)$ approaches either +1 or -1 ,

P3) $\|q(t)\|_{2}$ approaches 0.

PROOF: Clear from the remarks above.

q.e.d.

Property 4 states that the control law (12) guarantees (under rather mild conditions) convergence of the dynamical system (1)-(3) to $\omega=0, q=0$, $q_{4}= \pm-1$. Interestingly, this convergence guarantee can be made for any constant gain $k$, both, for $k>0$ and $k<0$. However, Property 4 does not provide any help in deciding which sign to pick for $k$ to perform a given reorientation most effectively. As a rule-of-thumb strategy one may want to apply the logic to pick $k$ such that $q_{4} \rightarrow+1$ if $q_{4}\left(t_{0}\right) \geq 0$, and, vice versa. To enable such a choice, the next result addresses the question to which value $(+1$ or -1) $q_{4}$ can be expected to converge as a function of the sign chosen for the gain $k$.

Property 5 Assume the gains $k$ and $c$ are constant with $c<0$ and $k \neq 0$. Then the eigenvalues of the system obtained from linearizing the equations of motion (1)-(3) with the control law (12) about $\omega=0, q=0$, has the following properties:

P1) The eigenvalue associated with the $q_{4}$ component is always zero.

P2) If $k q_{4}>0$, then all eigenvalues associated with the $\omega$ and q-components have negative real parts.

P3) If $k q_{4}<0$, then three eigenvalues associated with the $\omega$ and q-components have negative real parts, and three have positive real parts.

Proof: Linearizing the equations of motion (1)-(3) with the control law (12) about $\omega=0, q=0$, leads to the linear dynamical system

$$
\dot{\delta} x=A \delta x
$$

where $x^{T}=\left[\delta \omega^{T}, \delta q^{T}, \delta q_{4}\right]$ and

$$
A=\left(\begin{array}{rrrrrrr}
-c & 0 & 0 & -k & 0 & 0 & 0 \\
0 & -c & 0 & 0 & -k & 0 & 0 \\
0 & 0 & -c & 0 & 0 & -k & 0 \\
\frac{q_{4}}{2} & 0 & 0 & 0 & 0 & 0 & 0 \\
0 & \frac{q_{4}}{2} & 0 & 0 & 0 & 0 & 0 \\
0 & 0 & \frac{q_{4}}{2} & 0 & 0 & 0 & 0 \\
0 & 0 & 0 & 0 & 0 & 0 & 0
\end{array}\right)
$$


Obviously, the eigenvalue $\lambda_{7}$, associated with $\delta q_{4}$ is always zero. Using a determinant relation for partitioned matrices [15], it can be shown that the remaining matrix $\bar{A}$, obtained by deleting the last row and last column of $A$, has the eigenvalues

$$
\begin{aligned}
& \lambda_{1}=\lambda_{2}=\lambda_{3}=\frac{-2 c-\sqrt{4 c^{2}-8 k q_{4}}}{4} \\
& \lambda_{4}=\lambda_{5}=\lambda_{6}=\frac{-2 c+\sqrt{4 c^{2}-8 k q_{4}}}{4}
\end{aligned}
$$

Noting that $2 c>\sqrt{4 c^{2}-8 k q_{4}}$ if $k q_{4}>0$, and $2 c<\sqrt{4 c^{2}-8 k q_{4}}$ if $k q_{4}<0$, the statement of Property 5 follows immediately from (56), (57).

q.e.d.

Clearly, from the results of Property 5 it can be expected that under the influence of external perturbations $q_{4}$ will always converge to +1 if $k$ is picked positive, and, vice versa, $q_{4}$ will always converge to -1 if $k$ is picked negative.

These remarks, however, should be understood only as educated guesses. Note that $k$ and $c$ are assumed constant in Property 5, while $c$ was assumed possibly non-constant in Properties 3 and 4.

\section{Slew Rate Constraint}

To address the slew rate constraint, we will make use of the following intuitive logic: The solution $x(t)$ of an ordinary differential equation $\dot{x}=f(x, t)$ is guaranteed to satisfy the constraint $g(x, t) \leq 0$ if the functional dependence of $f$ and $g$ on $x$ and $t$ is such that the slope of $g$ goes to zero whenever $g$ approaches zero (from below). The associated lemmas summarizing the relevant results are given in the Appendix. In the following these results will be applied to the slew rate constraints (10), (11).

\section{Two-Norm Constraint}

For the slew rate constraint (10) we have the following result.

Property 6 Consider the system (1)-(3) with the control law (12) and the slew rate constraint (10) with $\omega_{\text {max }}>0$. Assume that the functional dependence of the gains $k$ and $c$ on their arguments $\omega, q$, $q_{4}$, and $t$ is such that

$$
c \geq-k \frac{\omega^{T} q}{\omega^{T} \omega}
$$

is satisfied whenever

$$
\|\omega\|_{2} \geq \omega_{\max }
$$

Then the slew rate constraint (10), i.e.

$$
\|\omega(t)\|_{2} \leq \omega_{\max },
$$

is guaranteed to remain satisfied for all times $t \geq t_{0}$ if it is satisfied at the initial time $t_{0}$.

Proof: Noting that

$$
\begin{aligned}
\frac{d}{d t}\left(\omega^{T} \omega\right) & =2 \omega^{T} \dot{\omega} \\
& =2 \omega^{T}(-k q-c \omega) \\
& =-2 k \omega^{T} q-2 c \omega^{T} \omega
\end{aligned}
$$

the statement of Property 6 follows from Lemma 1 in the Appendix.

$$
\text { q.e.d. }
$$

Intuitively, it can be expected that Property 6 remains satisfied if the inequality sign in (59) is replaced by an equality sign. With the help of Lemma (2) it can be shown that this is in fact correct if $k\left(\omega, q, q_{4}, t\right)$ and $c\left(\omega, q, q_{4}, t\right)$ also satisfy the conditions stated in Property 1 . We have the following result.

Property 7 Consider the system (1)-(3) with the control law (12) and the slew rate constraint (10) with $\omega_{\text {max }}>0$. Assume the gains $k\left(\omega, q, q_{4}, t\right)$ and $c\left(\omega, q, q_{4}, t\right)$ are Lipschitz-bounded with respect to $\omega, q, q_{4}$, and piecewise continuous with respect to $t$. Additionally, assume that $k$ and $c$ are such that

$$
c \geq-k \frac{\omega^{T} q}{\omega^{T} \omega}
$$

is satisfied whenever

$$
\|\omega\|_{2}=\omega_{\max }
$$

Then the slew rate constraint (10), i.e.

$$
\|\omega(t)\|_{2} \leq \omega_{\max },
$$

is guaranteed to remain satisfied for all times $t \geq t_{0}$ if it is satisfied at the initial time $t_{0}$.

Proof: Follows from Lemma 2 in the Appendix.

$$
\text { q.e.d. }
$$

Now assume $c$ and $k$ are such that conditions (58), (59) (or (61), (62)) are satisfied. Then it is immediately clear that the same conditions remain satisfied, if $c$ is divided in half, or if $k$ is multiplied by two. In fact, the following general result can be easily verified. 
Remark 1 Assume $c$ and $k$ are such that Property 6 (or Property 7) is satisfied. Let $\lambda_{1}>0$, $\lambda_{2}>0$ be positive constants. Then, Property 6 (or Property 7) remains satisfied if we replace

$$
\begin{array}{ll}
c & \rightarrow c \lambda_{1} \\
k & \rightarrow \quad k \lambda_{2} \\
\omega_{\max } & \rightarrow \omega_{\max } \frac{\lambda_{2}}{\lambda_{1}}
\end{array}
$$

From Remark 1 it follows that, by picking $c$ larger (or $k$ smaller) than required by the conditions of Properties 6 or 7 , the maximum attainable slew rate is reduced to values smaller than $\omega_{\max }$. In practice, this may represent an undesired performance degradation, and it may hence be a design goal to satisfy conditions (58) / (61) only marginally, i.e. with equality rather than strict inequality.

However, simply replacing the inequality signs in conditions (58), (61) by equality signs would lead to controller gains that maintain $\|\omega(t)\|_{2}$ at a constant value for all times after $\|\omega\|_{2}=\omega_{\max }$ is reached. Obviously, this is not desirable. Formally, such an approach would also violate condition (17), which is required in our proof of global stability (see Property 4).

A sensible strategy that leads to a controller which satisfies Properties 1, 2, 4 of this paper and marginally satisfies the slew rate constraint (10), is to pick a constant gain $k>0$, and to enforce conditions (58), (61) of Property 6 or 7 with strict equality, but to override this constraint (and instead enforce (58), (61) with strict inequality) whenever this is required by at least one of the conditions of Properties $1,2,4$. It can be easily verified that all conditions of Properties 1, 2, 4 can be satisfied this way. The approach leads to a constant gain $k$ and to a piecewise defined, continuous gain function $c$.

A convenient simplification of the conditions of Properties 6 and 7 is as follows.

Remark 2 The conditions (58), (59) of Property 6 (conditions (61), (62)) of Property 7) are guaranteed to be satisfied if the functional dependence of $k$ and $c$ on their arguments $\omega, q, q_{4}$, and $t$ is such that

$$
c=|k| \frac{\|q\|_{2}}{\omega_{\max }}
$$

whenever $\|\omega\|_{2} \geq \omega_{\max }$ (whenever $\|\omega\|_{2}=\omega_{\max }$ ).

For the special case where $-k \omega^{T} q=|k|\|\omega\|_{2}\|q\|_{2}$, the right-hand sides of conditions (63), (58) (conditions (63), (61)) are identical. In this case, condition (58) (condition (61)) is satisfied only marginally and the slew rate constraint (10) can be satisfied with equality.
To see this, note that for $\|\omega\|_{2} \geq \omega_{\max }$

$$
c=|k| \frac{\|q\|_{2}}{\omega_{\max }} \geq|k| \frac{\|q\|_{2}}{\|\omega\|_{2}} \geq|k| \frac{\left|\omega^{T} q\right|}{\|\omega\|_{2}^{2}} \geq-k \frac{\omega^{T} q}{\omega^{T} \omega}
$$

\section{$\underline{\text { Infinity-Norm Constraint }}$}

In many practical applications, the slew rate constraint has to be enforced in terms of the infinity norm (11) in favor over the two-norm (10). Clearly, the slew rate constraint (11) can be equivalently expressed in terms of the three scalar conditions

$$
\omega_{i}(t) \leq \omega_{\max }, \quad i=1,2,3 .
$$

Applying the findings of Lemma 1 in the Appendix to any one of the components in (64) we obtain the following result.

Property 8 Consider the system (1)-(3) with the control law (12) and a slew rate constraint of the form (64) with fixed $i \in\{1,2,3\}$ and $\omega_{\max }>0$. Assume that the functional dependence of the gains $k$ and $c$ on their arguments $\omega, q, q_{4}$, and $t$ is such that

$$
c \geq-k \frac{q_{i}}{\omega_{i}}
$$

is satisfied whenever

$$
\left|\omega_{i}\right| \geq \omega_{\max }
$$

Then the slew rate constraint

$$
\left|\omega_{i}(t)\right| \leq \omega_{\max }
$$

is guaranteed to remain satisfied for all times $t \geq t_{0}$ if it is satisfied at the initial time $t_{0}$.

Proof: Noting that

$$
\dot{\omega}_{i}=-c \omega_{i}-k q_{i}
$$

(see equation (13)), the statement of Property 8 follows from applying Lemma 1 in the Appendix two times, namely to the constraints $\omega_{i}-\omega_{\max } \leq 0$ and $-\omega_{i}-\omega_{\max } \leq 0$.

q.e.d.

In complete analogy to Property 7 the statement of Property 8 can be strengthened by replacing the equality sign in (66) through an equality sign. Explicitly, we have the following result. 
Property 9 Consider the system (1)-(3) with the control law (12) and a slew rate constraint of the form (64) with fixed $i \in\{1,2,3\}$ and $\omega_{\text {max }}>0$. Assume the gains $k\left(\omega, q, q_{4}, t\right)$ and $c\left(\omega, q, q_{4}, t\right)$ are Lipschitz-bounded with respect to $\omega, q, q_{4}$, and piecewise continuous with respect to $t$. Additionally, assume that $k$ and $c$ are such that

$$
c \geq-k \frac{q_{i}}{\omega_{i}}
$$

is satisfied whenever

$$
\left|\omega_{i}\right|=\omega_{\max }
$$

Then the slew rate constraint

$$
\left|\omega_{i}(t)\right| \leq \omega_{\max }
$$

is guaranteed to remain satisfied for all times $t \geq t_{0}$ if it is satisfied at the initial time $t_{0}$.

Proof: Follows from Lemma 2 in the Appendix.

$$
\text { q.e.d. }
$$

Properties 8 and 9 apply for each component of the three constraints $i=1,2,3$ in (64) individually. Any combination of these constraints is satisfied if $k$ and $c$ are such that conditions $(65),(66)$, in the case of Property 8, and conditions (68), (69), in the case of Property 9, are satisfied for the appropriate combination of indices $i$.

In complete analogy to Remark 1 we have:

Remark 3 Assume $c$ and $k$ are such that Property 8 (or Property 9) is satisfied. Let $\lambda_{1}>0$, $\lambda_{2}>0$ be positive constants. Then, Property 8 (or Property 9) remains satisfied if we replace

$$
\begin{aligned}
& c \quad \rightarrow c \lambda_{1} \\
& k \quad \rightarrow \quad k \lambda_{2} \\
& \omega_{\max } \rightarrow \omega_{\max } \frac{\lambda_{2}}{\lambda_{1}}
\end{aligned}
$$

In complete analogy to the conclusions drawn from Remark 1 it follows from Remark 3 that, by picking $c$ smaller (or $k$ larger) than required by the conditions of Properties 8 or 9 , the maximum attainable slew rate is reduced to values smaller than $\omega_{\max }$. In practice, this may represent an undesired performance degradation, and it may hence be a design goal to satisfy conditions (65) / (68) only marginally, i.e. with equality rather than strict inequality. From the same arguments as those used in the paragraphs following Remark 1 it follows, as in the previous section, that it is not sensible to blindly enforce conditions $(65) /(68)$ with strict equality throughout.

In analogy to Remark 2, a convenient simplification of the conditions of Properties 6 and 7 can be given as follows.
Remark 4 The conditions (65), (66) of Property 8 (conditions (68), (69) of Property 9) are guaranteed to be satisfied if the functional dependence of $k$ and $c$ on their arguments $\omega, q, q_{4}$, and $t$ is such that

$$
c=|k| \frac{\left|q_{i}\right|}{\omega_{\max }} .
$$

whenever $\left|\omega_{i}\right| \geq \omega_{\max }$ (whenever $\left|\omega_{i}\right|=\omega_{\max }$ ).

\section{$\underline{\text { Summary and Conclusions }}$}

We have analyzed a nonlinear feedback approach to the problem of reorienting a rigid spacecraft from arbitrary initial conditions to prescribed final conditions with zero angular velocity. The control law, which is derived from feedback linearization, is based on quaternion feedback and leaves the user to choose two gains as functions of position, angular rate, and time. Under mild conditions on these gains it is shown that the control law has the following properties: 1) rest-to-rest boundary conditions result in a principal axis rotation, 2) for arbitrary initial conditions, the rotational motion perpendicular to the principal axis decays exponentially, and 3 ) the prescribed final states are guaranteed to be reached asymptotically in time. Additionally, the paper analyzes slew rate constraints, both, in terms of the two-norm and the infinity-norm. For both cases, conditions on the controller gains are derived that guarantee satisfaction of the slew rate limit at all times.

In summary, the paper provides the design engineer with a general nonlinear control law with appealing closed-loop properties. Global asymptotic stability and satisfaction of slew rate constraints can be guaranteed by observing the simple rules on the controller gain design derived in this paper.

\section{Acknowledgements}

The authors would like to thank Renjith R. Kumar and Min Qu of Analytical Mechanics Associates, Inc., Hampton, Virginia, for useful discussions on this subject.

\section{$\underline{\text { Appendix }}$}

Lemma 1 Consider the initial value problem

$$
\dot{x}=f(x, t), \quad x\left(t_{0}\right)=x_{0}
$$

and the state constraint

$$
g(x, t) \leq 0
$$


Assume that the functional dependence of the functions $f$ and $g$ on their arguments $x$ and $t$ is such that

$$
\frac{\partial g(x, t)}{\partial x} f(x, t)+\frac{\partial g(x, t)}{\partial t} \leq 0
$$

is satisfied for all $x$ and that are such that

$$
g(x, t) \geq 0 .
$$

Then the constraint (72) is guaranteed to remain satisfied for all times $t \geq t_{0}$ along the solution of (71) if (72) is satisfied at the initial time $t_{0}$, i.e., if $g\left(x_{0}, t_{0}\right) \leq 0$.

Proof: We have

$$
\frac{d}{d t} g(x, t)=\frac{\partial g(x, t)}{\partial x} f(x, t)+\frac{\partial g(x, t)}{\partial t}
$$

Hence, conditions (73), (74) imply that $\frac{d g}{d t} \leq 0$ whenever $g \geq 0$. Hence, with $g\left(x_{0}, t_{0}\right) \leq 0$, it is clear that (72) remains satisfied for all times $t \geq t_{0}$.

q.e.d.

Intuitively, it can be expected that Lemma 1 remains satisfied if the inequality sign in (74) is replaced by an equality sign. It can be shown that this is in fact correct if the functions $f(x, t)$ and $g(x, t)$ are sufficiently "well-behaved". Explicitly, we have the following result.

Lemma 2 Consider the initial value problem (71) and the state constraint (72). Assume that $g$ is differentiable and that $f(x, t), \frac{\partial g(x, t)}{\partial x}$, and $\frac{\partial g(x, t)}{\partial t}$ are Lipschitz-bounded with respect to $x$, and piecewise continuous with respect to $t$. Additionally, assume that the functional dependence of the functions $f$ and $g$ on their arguments $x$ and $t$ is such that

$$
\frac{\partial g(x, t)}{\partial x} f(x, t)+\frac{\partial g(x, t)}{\partial t} \leq 0
$$

is satisfied for all $x$ and that are such that

$$
g(x, t)=0 .
$$

Then the constraint (72) is guaranteed to remain satisfied for all times $t \geq t_{0}$ along the solution of (71) if (72) is satisfied at the initial time $t_{0}$, i.e., if $g\left(x_{0}, t_{0}\right) \leq 0$.

Proof: Define

$$
h(x, t)=\frac{\partial g(x, t)}{\partial x} f(x, t)+\frac{\partial g(x, t)}{\partial t}
$$

and consider the initial value problem

$$
\left[\begin{array}{l}
\dot{x} \\
\dot{y}
\end{array}\right]=\left[\begin{array}{c}
f(x, t) \\
\bar{h}(x, t)
\end{array}\right], \quad\left[\begin{array}{l}
x\left(t_{0}\right) \\
y\left(t_{0}\right)
\end{array}\right]=\left[\begin{array}{c}
x_{0} \\
g\left(x_{0}, t_{0}\right)
\end{array}\right]
$$

where

$$
\bar{h}(x, y, t)= \begin{cases}h(x, t) & \text { if } \quad y \leq 0 \\ 0 & \text { if } y>0\end{cases}
$$

Let the solution of (79) be denoted by superscript hat. Using Lemma 1 it can be shown that the solution of the initial value problem (79) satisfies the constraint $y \leq 0$, i.e. we have $\hat{y}(t) \leq 0$, if $g\left(x_{0}, t_{0}\right) \leq 0$. Hence $\hat{x}(t), \hat{y}(t)$ also furnish a solution to the initial value problem

$$
\left[\begin{array}{l}
\dot{x} \\
\dot{y}
\end{array}\right]=\left[\begin{array}{l}
f(x, t) \\
h(x, t)
\end{array}\right], \quad\left[\begin{array}{l}
x\left(t_{0}\right) \\
y\left(t_{0}\right)
\end{array}\right]=\left[\begin{array}{c}
x_{0} \\
g\left(x_{0}, t_{0}\right)
\end{array}\right]
$$

if $g\left(x_{0}, t_{0}\right) \leq 0$. Clearly, the functions $f, \frac{\partial g}{\partial x}$, and $\frac{\partial g}{\partial t}$ in $(81)$ are such that it can be guaranteed that the solution to (81) is unique (see the Appendix I of [13]). Together with the results obtained above, this implies that the unique solution to (81) satisfies $y(t) \leq 0$. Noting that the $y$-component in $(81)$ can be integrated analytically to yield $y(t)=g(x(t), t)$ proves the statement of the lemma.

q.e.d.

Remark 5 The statement of Lemma 2 can be strengthened by replacing "piecewise continuous with respect to time t" through "measurable with respect to time $t . "$

Remark 6 The assumption of Lipschitz boundedness in Lemma 2 is, in fact, necessary and cannot be weakened.

For example, consider the initial value problem

$$
\dot{x}=\sqrt[3]{x^{2}}, \quad x\left(t_{0}\right)=x_{0}, \text { with } t_{0}=x_{0}=-1
$$

and the constraint

$$
x \leq 0
$$

The solution $x(t)$ of (82) obviously satisfies $x\left(t_{0}\right) \leq 0$ and the right-hand side of the differential equations in (82) are such that $\dot{x} \leq 0$ whenever $x=0$. However, it can be easily verified that

$$
x(t)=t^{3}
$$

furnishes a solution to (82), which obviously violates $x \leq 0$ for $t>0$. In fact, the right-hand side of the differential equation in (82) is not Lipschitz bounded at $x=0$, and it can be verified that

$$
x(t)= \begin{cases}t^{3} & \text { for } t \leq 0 \\ 0 & \text { for } t>0\end{cases}
$$

represents an alternative solution to (82). 


\section{References}

[1] Bilimoria, K.D. and Wie, B. "Minimum-Time Large-Angle Reorientation of a Rigid Spacecraft", AIAA Guidance, Navigation and Control Conference, Portland, Oregon, Paper No. 90-3486, August 1990.

[2] Chowdhry, R.S. and Cliff, E.M., "Optimal Rigid Body Reorientation Problem", AIAA Guidance, Navigation and Control Conference, Portland, Oregon, Paper No: 90-3485, August 1990.

[3] Junkins, J.L. and Turner, J.P., "Optimal Spacecraft Rotational Maneuvers", Elsevier Scientific Publishing, New York, 1985.

[4] Junkins, J.L. and Turner, J.P., "Optimal Continuous Torque Attitude Maneuvers", Journal of Guidance and Control, Vol.3, No.3, pp 210217, May-June 1980.

[5] Mortensen, R.E., “A globally Stable Linear Attitude Regulator", Internatinal Journal of Control, Vol. 8, No. 3, pp. 297-302.

[6] Farrenkopf, R. L., "Optimal Open Loop Maneuver Profiles for Flexible Spacecraft," Journal of Guidance, Control, and Dynamics, Vol. 2, No. 6, pp. 491-498.

[7] Turner, J. D., Junkins, J. L., "Optimal Large Angle Single Axis Maneuvers of Flexible Spacecraft," Journal of Guidance, Control, and Dynamics, Vol. 3, No. 5, pp. 578-585.

[8] Singh, G., Kabamba, P. T., and McClamroch, N. H., "Planar Time Optimal Slewing Maneuvers of Flexible Spacecraft," Journal of Guidance, Control, and Dynamics, Vol. 12, No. 1, pp. $71-81$.

[9] Wie, B. , Weiss H., and Araposthathis, A., "Quaternion Feedback Control for Spacecraft Eigenaxis Rotations," Journal of Guidance, Control, and Dynamics, Vol. 12, No. 3, pp. 759775 .

[10] Bauer, F. H., Femiano, M. D., and Mosier, G. E., "Attitude Control System Conceptual Design for the X-Ray Timing Explorer," Proceedings of the AIAA Guidance, Navigation, and Control Conference, AIAA, Washington DC, 1992, pp. 236-249.

[11] Wie, B. and Barba, P., "Quaternion Feedback for Spacecraft Large Angle Maneuvers,"
Journal of Guidance, Control, and Dynamics, Vol. 8, No. 3, pp. 360-365.

[12] Wie, B. and Lu, J., "Feedback Control Logic for Spacecraft Eigenaxis Rotations Under Slew Rate and Control Constraints," Journal of Guidance, Control, and Dynamics, Vol. 18, No. 6, pp. 1372-1379.

[13] Lee, E.B., and Markus, L., "Foundations of Optimal Control Theory," Robert E. Krieger Publishing Company, Malabar, Florida, 1986.

[14] Bruce D. Craven, "Lebesgue Measure and Integral," Pitman Publishing Inc., Boston, 1982, Section 5.1.

[15] Beyer, W.H., CRC Standard Mathematical Tables, 26th edition, 1981, CRC Press, Inc., Boca Ratan, FL, p.35. 